\title{
Daily Consumption of Angelica Keiskei Juice Attenuated Hyperlipidaemia and Hepatic Steatosis 范擩 Caused by Western Diet in C57BL/6J Mice
}

\author{
Bora Kwon ${ }^{1}$, Soo Yeon Park ${ }^{1}$, Yeni Lim¹, Byung Hwa Jung ${ }^{2}$, Hyun A Oh ${ }^{2}$, Ji Yeon Kim³ and Oran Kwon*1 \\ ${ }^{1}$ Department of Nutritional Science and Food Management, Ewha Womans University, Republic of Korea \\ ${ }^{2}$ Molecular Recognition Research Center, Korea Institute of Science and Technology, Republic of Korea
}

${ }^{3}$ Department of Food Science and Technology, Seoul National University of Science and Technology, Republic of Korea

Received: 監: October 08, 2018; Published: 睯: October 24, 2018

*Corresponding author: Oran Kwon, Department of Nutritional Science and Food Management, Ewha Womans University, 52 Ewhayeodaegil, Seodaemun-gu, Seoul 03760, Republic of Korea

\begin{abstract}
Background/Objectives: Given that western diet (WD) intake has negative impact on development of non-alcoholic fatty liver disease (NAFLD), the protective effects of Angelica keiskei juice (AK) against hyperlipidemia and hepatic steatosis were investigated in WD-fed C57BL/6J mice.

Materials and Methods: Animals were assigned into four groups ( $n=8$ /group) to receive normal diet + vehicle, WD + vehicle, WD + low AK (2 $\mathrm{g} / \mathrm{kg}$ body weight), and WD + high AK ( $4 \mathrm{~g} / \mathrm{kg}$ body weight) for 18 weeks. Biochemical analysis was performed for lipid profile in plasma; histological assessment for steatosis, inflammation, and tissue damage in the liver and intestine; and qPCR analysis for intestinal lipoprotein lipase and hepatic endoplasmic reticulum (ER)/oxidative stress markers.

Results: AK significantly attenuated WD-induced increases in plasma triglyceride and very low density lipoprotein ( $\mathrm{P}=0.001$ for both), and hepatic triglyceride $(\mathrm{P}<0.001)$. Particularly, high-dose AK was effective in maintaining intestinal barrier integrity and increasing mRNA expression of hepatic nuclear factor erythroid 2-related factor $2(\mathrm{P}=0.003)$. It also tended to modify mRNA expressions of intestinal lipoprotein lipase ( $\mathrm{P}=0.004)$, and hepatic carnitine palmitoyl transferase $1 \alpha(\mathrm{P}=0.038)$, endoplasmic reticulum nuclei-1 $(\mathrm{P}=0.014)$, and NAD $(\mathrm{P}) \mathrm{H}$ : quinone oxidoreductase 1 $(\mathrm{P}=0.047)$. Conclusions: These findings suggest that daily consumption of AK juice may have potentials to prevent WD-induced NAFLD development through mitigating intestinal barrier damage, intestinal lipid absorption, and hepatic ER oxidative stress.
\end{abstract}

Keywords: Angelica Keiskei Juice; Western Diet; Hyperlipidemia; Non-Alcoholic Fatty Liver Disease; C57BL/6J Mice

Abbreviations: WD: Western Diet; NAFLD: Non-Alcoholic Fatty Liver Disease; AK: Angelica Keiskei Juice; ER: Endoplasmic Reticulum; TG: Triglyceride; HESI: Heated Electrospray Ionization; NC: Normal Diet, WC: WD Control; EDTA: Ethylene Diamine Tetra-Acetic Acid; IACUC: Institutional Animal Care And Use Committee; TC: Total Cholesterol; HDL: High-Density Lipoprotein Cholesterol; LDL: Low Density Lipoprotein; VLDL: Very LowDensity Lipoprotein; H\&E: Haematoxylin and Eosin; UPR: Unfolded Protein Response

\section{Introduction}

Angelica keiskei, commonly known as Ashitaba, is a cold hardy perennial plant in the genus of Angelica. It has been traditionally used as a tonic to restore vitality [1]. Since 1990s, scientific work has begun to explore the chemo protective effects of chalcone, a bioactive component isolated from Angelica keiskei, both in vitro and in vivo (animals) [2]. In the early 2000s, investigations were expanded to the metabolic effects of Angelica keiskei and/or its bioactives (chalcone and coumarins) including lipid metabolism [3], insulin resistance [4], arterial relaxation [5], oxidative stress
[6], inflammation [7], and chemical-induced hepatotoxicity [8,9]. The typical Western diet (WD) is generally characterized by an increase in total fat, saturated fat, and sugars, but a decrease in fiber. The bulk of ingested sugar is rapidly metabolized into glucose6phosphate which is trapped inside cells. This pathway enables rapid conversion of excess glucose to lipid, resulting in elevated blood lipid levels and exacerbation of triglyceride (TG) storage in the liver. Excess hepatic TG accumulation sensitizes the liver to induce oxidative stress, insulin resistance, and inflammation, eventually leading to hepatocellular damage and death [10]. 
In addition, high amounts of total fat and saturated fat may alter intestinal barrier integrity and result in direct exposure of intestinal endotoxins to the liver through portal vein $[11,12]$. Subsequently, these endotoxins can stimulate liver-resident macrophages called Kupffer cells, thereby leading to oxidative stress and inflammation related hepatic damage [13,14]. Furthermore, an additive effect of high-fat and high-sucrose diet on the development of hepatic steatosis was confirmed in mice by Ishimato et al. [15]. Although the best strategy to prevent has not been defined yet, experimental evidence has suggested that plant foods and their constituents might be useful for controlling the onset and progression of WDinduced non-alcoholic fatty liver disease (NAFLD) [16,17]. Based on these findings, we hypothesized that daily consumption of Angelica keiskei might have an impact on reducing WD-induced hyperlipidemia and NAFLD development through enhancing lipid metabolism as well as suppressing the oxidative stress. To test this hypothesis, we performed an animal study using a WD-induced NAFLD mice model. Furthermore, to gain a better understanding on the underlying mechanisms, we analysed histological sections of intestine and liver in relation to intestinal barrier integrity and hepatic steatosis and examined gene expressions associated with oxidative stress in mitochondria and microsome fractions of the liver. To the best of our knowledge, this is the first study that assesses the preventive effect of Angelica keiskei against

WD-induced NAFLD.

\section{Materials and Methods}

\section{Preparation of Angelica Keiskei Juice Powder}

Angelica keiskei juice powder (AK) was provided by Daesang Corp. (Seoul, Korea). Briefly, leaves of Angelica keiskei were grounded, squeezed, and freeze-dried to powder for use in this study. Chemical characteristics of AK were analysed using an Ultimate 3000 UPLC system from Thermo Fisher Scienctific ${ }^{\text {TM }}$ (San Jose, CA, USA) and an LTQ Orbitrap Velos Pro ${ }^{\mathrm{TM}}$ system mass spectrometer (San Jose, CA, USA). Samples were injected into a Waters ACQUITY® UPLC BEH C18 column $(2.1 \times 100 \mathrm{~mm}, 1.7 \mu \mathrm{m})$ at a temperature of $50{ }^{\circ} \mathrm{C}$. The gradient of elution was performed using mobile phase A $(0.1 \%$ formic acid in DW) and mobile phase B $(0.1 \%$ formic acid in methanol) at a flow rate of $0.4 \mathrm{~mL} / \mathrm{min}$. The gradient condition was regulated as follows: $100 \%$ of mobile phase A for $0-1 \mathrm{~min}$, a linear decrease of mobile phase A from 100 to $1 \%$ from 1-13min; and re-equilibration with $100 \%$ mobile phase A from $13-$ $14 \mathrm{~min}$. The injection volume of all samples was $5 \mu \mathrm{L}$. Temperature of the auto sampler was kept at $4{ }^{\circ} \mathrm{C}$. Heated electrospray ionization (HESI) source was used in both positive and negative modes and the capillary temperature was set at $320^{\circ} \mathrm{C}$. The spray voltage was $3.8 \mathrm{kV}$ and the S-lens RF level was $61 \%$. Nitrogen was used as both sheath gas and auxiliary gas. Xcalibur software was used for data analysis.

\section{Animals and Treatments}

Five-week-old male C57BL/6J mice (17-20g) were purchased from Jung-Ang Lab Animal (Seoul, Korea). Following acclimatization for one week, animals were randomly assigned into four experimental groups ( $n=8$ per group) to receive normal diet (NC),
WD control (WC), low-dose AK (2g/kg body weight) with WD (AL), and high-dose $\mathrm{AK}(4 \mathrm{~g} / \mathrm{kg}$ body weight) with WD (AH) for 18 weeks. Normal diet (AIN-93G; Research Diets, New Brunswick, NJ, USA) provided $64 \% \mathrm{kcal}$ carbohydrate and $16 \% \mathrm{kcal}$ fat, whereas Western diet (D12079B; Research Diets) provided 43\% kcal carbohydrate and $41 \% \mathrm{kcal}$ fat. AK juice powder was dissolved in distilled water and administered orally to mice once daily by gavage. NC and WC groups received the same amount of distilled water by gavage. At the end of experimental period, overnight-fasted mice were euthanized by carbon dioxide inhalation. Whole blood samples were collected from the abdominal aorta and transferred to ethylene diamine tetra-acetic acid (EDTA) tubes. Plasma was obtained after centrifugation at $2,760 \mathrm{rpm}$ for $10 \mathrm{~min}$ at $4{ }^{\circ} \mathrm{C}$ and frozen at $-80{ }^{\circ} \mathrm{C}$ prior to use. Intestine and liver were removed, weighed, and frozen at $-80{ }^{\circ} \mathrm{C}$ for biochemical and molecular analyses or preserved with phosphate-buffered formalin for histological observation. The experimental protocol was approved by the Institutional Animal Care and Use Committee (IACUC) of Ewha Womans University (No. 14-099). Animal care and handling were conducted in compliance with the National Research Council's Guide for the Care and Use of Laboratory Animals.

\section{Biochemical Assays}

Plasma TG, total cholesterol (TC) and high-density lipoprotein cholesterol (HDL) levels were measured using commercial colorimetric assay kits (Asan Pharm., Seoul, Korea). Plasma low density lipoprotein cholesterol (LDL) level was calculated using Friedewald equation [18]. Very low-density lipoprotein (VLDL) level was calculated by subtracting the sum of HDL and LDL values from the value of TC. Total lipid was extracted according to a modified Folch's method [19]. The lipid extract was dissolved in $5 \mathrm{ml}$ ethanol containing $0.5 \%$ Triton X-100 to determine TG level with a colorimetric assay kit (Asan Pharm).

\section{Histopathological Assessment}

Formalin-fixed liver and intestine tissues were dehydrated in various concentrations of ethyl alcohol, dealcoholized in xylene, embedded in paraffin, and cut into 5- $\mu$ m sections. Specimens were deparaffinized in xylene, rehydrated in a reverse-gradient series of ethyl alcohol, and stained with haematoxylin and eosin (H\&E). Steatosis, inflammation, and tissue damage were observed under a light microscope (Olympus, Tokyo, Japan).

\section{Preparation of Microsome And Mitochondria Fractions in the Liver}

Liver tissue was homogenized with 10 volumes of $1.15 \%$ potassium chloride buffer containing $10 \mathrm{mM}$ phosphate and $1 \mathrm{mM}$ EDTA (pH 7.4) followed by centrifugation at $1,900 \mathrm{rpm}$ for $10 \mathrm{~min}$ at $4{ }^{\circ} \mathrm{C}$. The supernatant was then centrifuged again at $10,000 \mathrm{rpm}$ for $20 \mathrm{~min}$ at $4^{\circ} \mathrm{C}$ to precipitate mitochondrial fraction. The resulting supernatant was centrifuged again at $34,000 \mathrm{rpm}$ for $1 \mathrm{~h}$ at $4{ }^{\circ} \mathrm{C}$ to precipitate microsomal fraction. Each pellet was suspended in $0.1 \mathrm{~mL}$ of buffer and stored at $-80^{\circ} \mathrm{C}$ until analysis. The mitochondria fraction was used for carnitine palmitoyl transferase $1 \alpha$ (CPT1 $\alpha$ ) analysis while the microsome fraction was used for endoplasmic reticulum nuclei-1 (ERN1), nuclear factor erythroid 2-related 
factor 2 (Nrf2), and NAD(P)H: quinone oxidoreductase 1 (NQO1) analyses.

\section{Quantitative Real-Time Reverse Transcription-Poly- merase Chain Reaction (qRT-PCR)}

Total RNAs were extracted from the liver, intestine, and hepatic microsome and mitochondria fractions using TRIzol Reagent (Invitrogen, Carlsbad, CA, USA). RNA concentration and quality were determined using BioSpec-nano spectrophotometer (Shimadzu, Kyoto, Japan). Single strand cDNA was constructed using a High Capacity cDNA Reverse Transcription Kit (Applied Biosystem, Foster City, CA, USA). Quantitative PCR was performed in a Step-OnePlus RT-PCR System (Applied Biosystem) using a TaqMan Universal PCR Master Mix Kit (Applied Biosystems). Primer sets for target genes were lipoprotein lipase (LPL, Mm01345523_m1), claudin-1
(CLDN1, Mm01342184_m1), occludin (OCLN, Mm00500912_m1), mucin 1 (MUC1, Mm00449604_m1), ERN1 (Mm00470233_m1), Nrf2 (Mm00477784_m1), NQ01 (Mm01253561_m1), CPT1 $\alpha$ (Mm01231183_m1), and $\beta$-actin (Mm02619580_g1). Relative amounts of these mRNAs were normalized to the amount of $\beta$-actin and calculated using comparative CT method. All data are expressed as a relative quantity to each control value.

\section{Statistical Analyses}

All results are presented as mean \pm SE. Statistical analyses were performed using the Statistical Analysis Systems package, version 9.4 (SAS Institute, Cary, NC, USA). Differences among groups were analysed by one-way analysis of variance (ANOVA) with post hoc Duncan's multiple comparison test. Statistical significance was considered at $\mathrm{P}<0.05$.

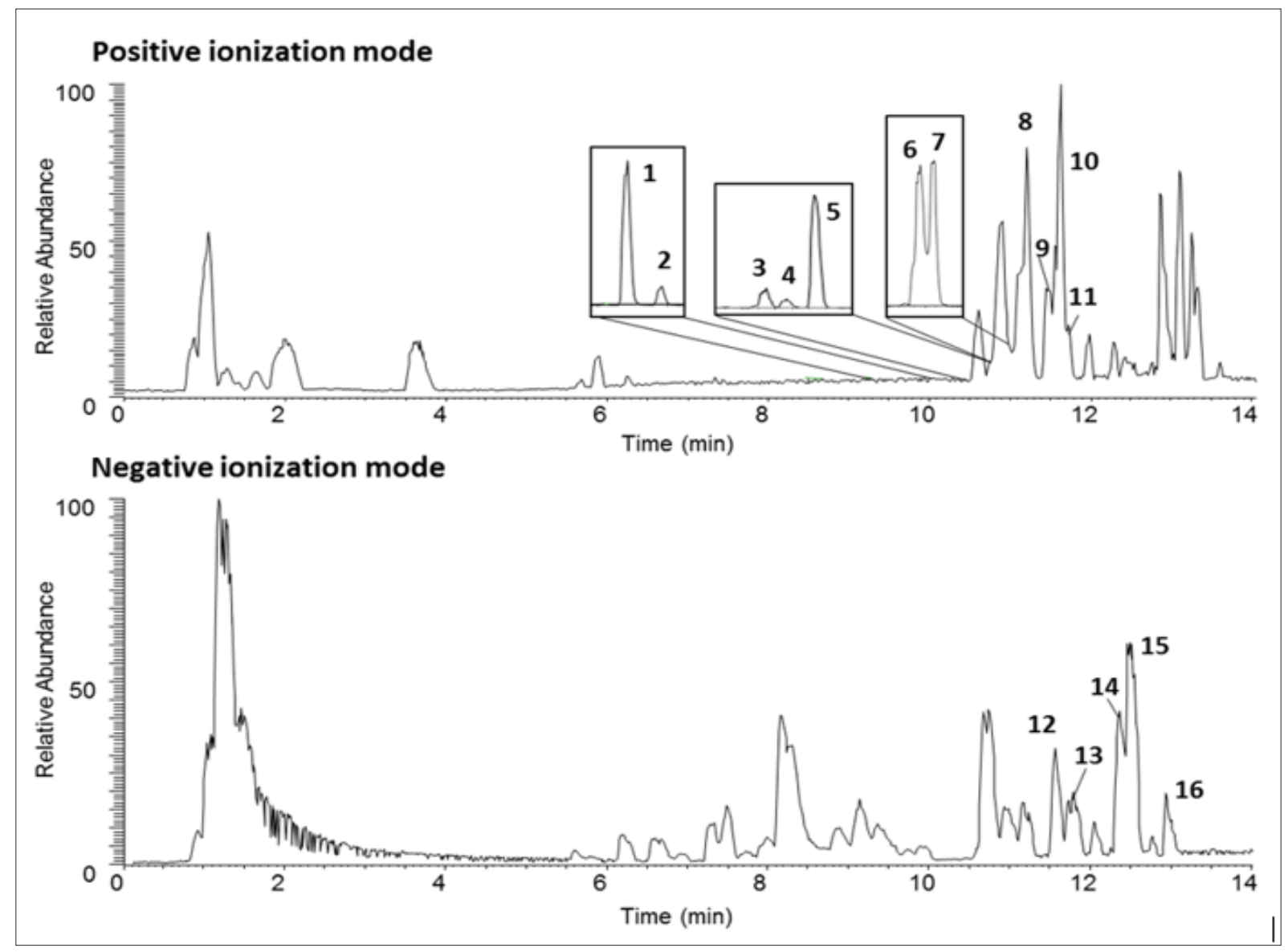

Figure 1: Representative UPLC-LTQ/MS base peak chromatograms of AK. The numbers on the chromatograms indicate each component described in the Table below. Chemical structures are provided in supporting information Table 1. AK, Angelica keiskei juice powder.

\section{Results}

\section{Chemical Composition of AK}

Chemical profile of AK is presented in Figure 1 and Table 1. A total of 16 signature components were identified by UPLC-LTQ/MS analysis, including 11 coumarins (8-methoxypsoralen, 5methoxy- psoralen, psoralen, isoimperatorin, demethylsuberosin, isopimpinellin, laserpitin, isolaserpitin, pteryxin, isopsoralen and selinidin) and 5 chalcones (xanthoangelol B, isobavachalcone, 4-hydroxyderricin, xanthoangelol and xanthoangelol F), compatible with results of a previous study by Kim et al. [20]. 
Table 1: Chemical characteristics of analysed using UPLC-LTQ/MS .

\begin{tabular}{|c|c|c|c|c|c|}
\hline No. & Components & RT & Adduct & $\mathbf{m} / \mathbf{z}$ & MS/MS \\
\hline & & & Coumarins & & \\
\hline 1 & 8-Methoxypsoralen & 9.44 & {$[\mathrm{M}+\mathrm{H}]+$} & 217.0499 & 201.9724 \\
\hline 2 & 5-Methoxypsoralen & 9.92 & {$[\mathrm{M}+\mathrm{H}]+$} & 217.0497 & 201.9595 \\
\hline 3 & Psoralen & 10.52 & {$[\mathrm{M}+\mathrm{H}]+$} & 187.0397 & 115.0760 \\
\hline 4 & Isoimperatorin & 10.65 & {$[M+H]+$} & 271.1077 & 147.0603 \\
\hline 5 & Demethylsuberosin & 10.65 & {$[\mathrm{M}+\mathrm{H}]+$} & 231.1199 & 213.0421 \\
\hline 6 & Isopimpinellin & 10.86 & {$[[\mathrm{M}+\mathrm{H}]+$} & 247.0655 & 217.0650 \\
\hline 7 & Laserpitin & 10.86 & {$[\mathrm{M}+\mathrm{H}]+$} & 367.0919 & 267.0192 \\
\hline 8 & Isolasepitin & 11.16 & {$[\mathrm{M}+\mathrm{Na}]+$} & 367.0918 & 349.0139 \\
\hline 9 & Pteryxin & 11.27 & {$[\mathrm{M}+\mathrm{Na}]+$} & 409.0956 & 391.0828 \\
\hline 10 & Isopsoralen & 11.47 & {$[\mathrm{M}+\mathrm{Na}]+$} & 187.0388 & 130.9519 \\
\hline \multirow[t]{2}{*}{11} & Selinidin & 11.49 & {$[M+H]+$} & 329.1483 & 311.1046 \\
\hline & & & Chalcones & & \\
\hline 12 & Xanthoangelol B & 11.68 & {$[\mathrm{M}-\mathrm{H}]-$} & 407.1807 & 119.0188 \\
\hline 13 & Isobavachalcone & 11.73 & {$[\mathrm{M}-\mathrm{H}]-$} & 323.1248 & 118.9545 \\
\hline 14 & 4-Hydroxyderrcin & 12.37 & {$[\mathrm{M}-\mathrm{H}]-$} & 337.1407 & 118.9917 \\
\hline 15 & Xanthoangelol & 12.51 & {$[\mathrm{M}-\mathrm{H}]-$} & 391.1870 & 118.9815 \\
\hline 16 & Xanthoangelol F & 12.97 & {$[\mathrm{M}-\mathrm{H}]-$} & 405.2021 & 118.9978 \\
\hline
\end{tabular}

\section{Effects of AK on Plasma and Hepatic Lipids}

The effects of AK on plasma and liver lipid levels are shown in Table 2. Compared to NC group, the WC group exhibited significant increases in plasma TG (109.20 \6.47 vs $142.11 \otimes 6.65)$, TC (107.27 $\otimes 2.68$ vs $152.92 \otimes 14.25)$, and VLDL (21.84 $\otimes 1.29$ vs $28.42 \otimes 1.33)$ levels, and hepatic total lipid (6.79 $\otimes 0.83$ vs $27.98 \otimes 3.17$ ), TG (13.58 $\otimes 0.75$ vs $23.64 \otimes 2.03)$, and TC ( $1.15 \otimes 0.11$ vs $2.43 \otimes 0.19$ ) levels. In contrast, daily administration of AK led to a significantly decreased levels in plasma TG $(\mathrm{P}=0.001)$ and VLDL $(\mathrm{P}=0.001)$ as well as those in hepatic total lipid $(\mathrm{P}<0.001)$ and $\mathrm{TG}(\mathrm{P}<0.001)$ in either AL or AH group compared with rats in WC group. However, AK administration did not affect plasma and hepatic TC levels. In consistent with these results, histology analysis revealed a severe hepatic steatosis in WC group as compared in NC group. However, AK administration at high dose inhibited fat droplet accumulation in liver tissue (Figure 2).

Table 2: Effects of AK on plasma and hepatic lipid levels.

\begin{tabular}{|c|c|c|c|c|}
\hline Variables & NC & WC & AL & AH \\
\hline $\begin{array}{c}\text { Plasma } \\
\text { TG (mg/dL) }\end{array}$ & $109.20 \pm 6.47^{\mathrm{b}}$ & $142.11 \pm 6.65^{\mathrm{a}}$ & $112.70 \pm 7.53^{\mathrm{b}}$ & $102.09 \pm 5.69^{\mathrm{b}}$ \\
\hline VLDL (mg/dL) & $21.84 \pm 1.29^{\mathrm{b}}$ & $28.42 \pm 1.33^{\mathrm{a}}$ & $22.54 \pm 1.51^{\mathrm{b}}$ & $20.42 \pm 1.14^{\mathrm{b}}$ \\
\hline TC (mg/dL) & $107.27 \pm 2.68$ & $152.92 \pm 14.25$ & $148.89 \pm 17.18$ & $151.33 \pm 12.24$ \\
\hline LDL (mg/dL) & $30.07 \pm 7.44$ & $42.35 \pm 11.06$ & $51.94 \pm 12.99$ & $52.41 \pm 13.06$ \\
\hline HDL (mg/dL) & $59.97 \pm 6.73$ & $75.23 \pm 13.01$ & $77.48 \pm 20.58$ & $78.60 \pm 16.53$ \\
\hline $\begin{array}{c}\text { Liver } \\
\text { Total lipid (mg/g) }\end{array}$ & $6.79 \pm 0.83^{\mathrm{c}}$ & $27.98 \pm 3.17^{\mathrm{a}}$ & $17.09 \pm 2.13 \mathrm{~b}$ & $12.35 \pm 1.81 \mathrm{~b}^{\mathrm{c}}$ \\
\hline TG (mg/g) & $13.58 \pm 0.75^{\mathrm{c}}$ & $23.64 \pm 2.03^{\mathrm{a}}$ & $18.16 \pm 0.53^{\mathrm{b}}$ & $15.75 \pm 0.34 \mathrm{~b}^{\mathrm{c}}$ \\
\hline TC (mg/g) & $1.15 \pm 0.11 \mathrm{~b}$ & $2.43 \pm 0.19^{\mathrm{a}}$ & $2.19 \pm 0.21^{\mathrm{a}}$ & $2.31 \pm 0.16^{\mathrm{a}}$ \\
\hline
\end{tabular}

Cite this article: Bora Kwon, Soo Yeon P, Yeni Lim, Byung Hwa J, Hyun A O , Ji Yeon K, Oran Kwon. Daily Consumption of Angelica Keiskei Juice Attenuated Hyperlipidaemia and Hepatic Steatosis Caused by Western Diet in C57BL/6J Mice. Biomed J Sci\&Tech Res 10(2)-2018. BJSTR. MS.ID.001933. DOI: 10.26717/ BJSTR.2018.10.001933. 

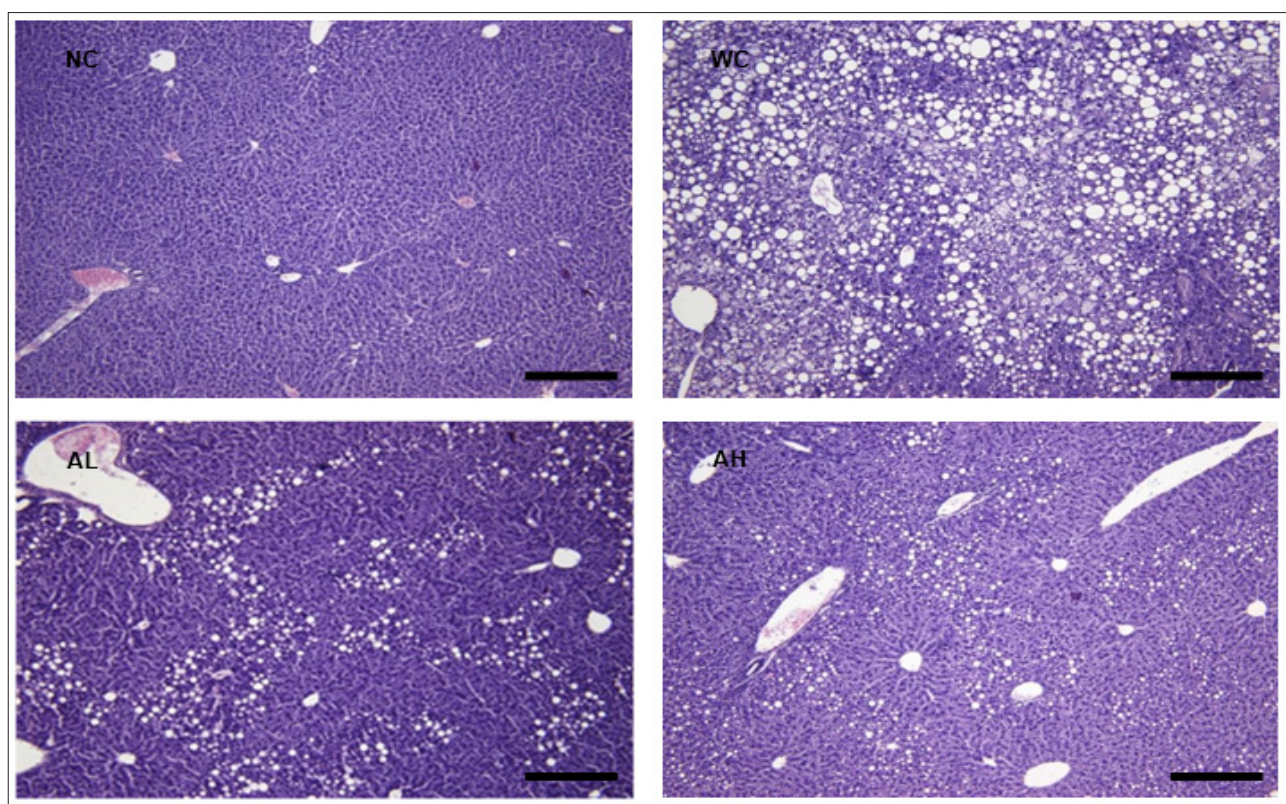

Figure 2: H\&E staining of the liver of C57BL/6J mice fed with WD for 12 weeks. AK, Angelica keiskei juice powder; NC, normal control; WC, western diet control; AL, low-dose AK (2g/kg BW) with WD; AH, high-dose AK (4g/kg BW) with WD.

\section{Effects of AK on Intestinal Barrier Integrity}

Histological examination of intestinal tissues is presented in Figure 3A, showing diffuse abnormalities of villi and surface epithelium, edema, and greater inflammation in WC group compared to NC group. However, AK administration at both doses almost completely prevented WD induced destruction of glandular epithelium. However, mRNA expressions of tight junction proteins (CLDN1 and OCLN) and mucin glycoprotein (MUC1) did not respond to either WD or AK (Figure 3B). Meanwhile, AH group showed a tendency of suppressing WD-induced increase in lipoprotein lipase (LPL) mRNA expression ( $\mathrm{P}=0.004)$ (Figure 3C).

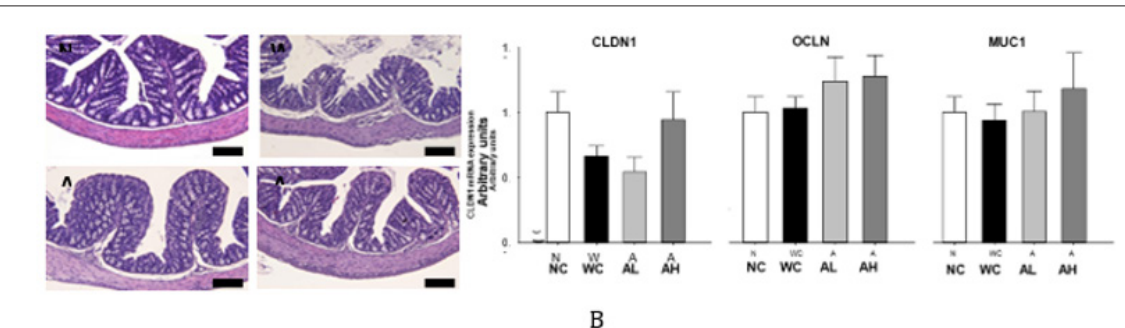

A. Gene expressions of hepatic CLDN1, OCLN, and MUC1 B. And mRNA expression of intestinal LPL

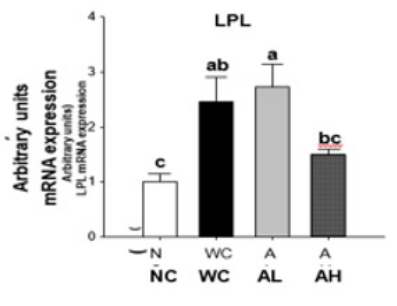

C. Of $\mathrm{C} 57 \mathrm{BL} / 6 \mathrm{~J}$ mice fed with WD and $\mathrm{AK}$ for 12 weeks.

Figure 3: H\&E staining AK, Angelica keiskei juice powder; CLDN1, claudin-1; OCLN, occludin; MUC1, mucin 1; LPL, lipoprotein lipase; NC, normal control; WC, western diet control; AL, low-dose AK (2g/kg BW) with WD; AH, high-dose AK $(4 \mathrm{~g} / \mathrm{kg} \mathrm{BW})$ with WD. Data are represented as means \pm SEM $(\mathrm{n}=8 /$ group$)$. Values with different alphabets in each panel are significantly different at $\mathrm{P}<0.05$ by Duncan's multiple comparison test.

Bar: $100 \mu \mathrm{m}$ for H\&E staining. 


\section{Effects of AK on ER Stress and Inflammation}

As indicators of ER stress and oxidative stress, we determined mRNA expressions of mitochondrial (CPT1 $\alpha$ ) and microsomal (ERN1, Nrf2, and NQO1) fractions in the liver. The WD induced significant decreases in the above four gene expressions, whilst high-dose AK administration significantly increased Nrf2 gene expression compared to that in WC group ( $\mathrm{P}=0.003)$. Also highdose AK administration showed a tendency of increasing CPT1 $\alpha$ $(\mathrm{P}=0.038)$, ERN1 $(\mathrm{P}=0.014)$, and NQO1 $(\mathrm{P}=0.047)$ gene expressions (Figure 4).

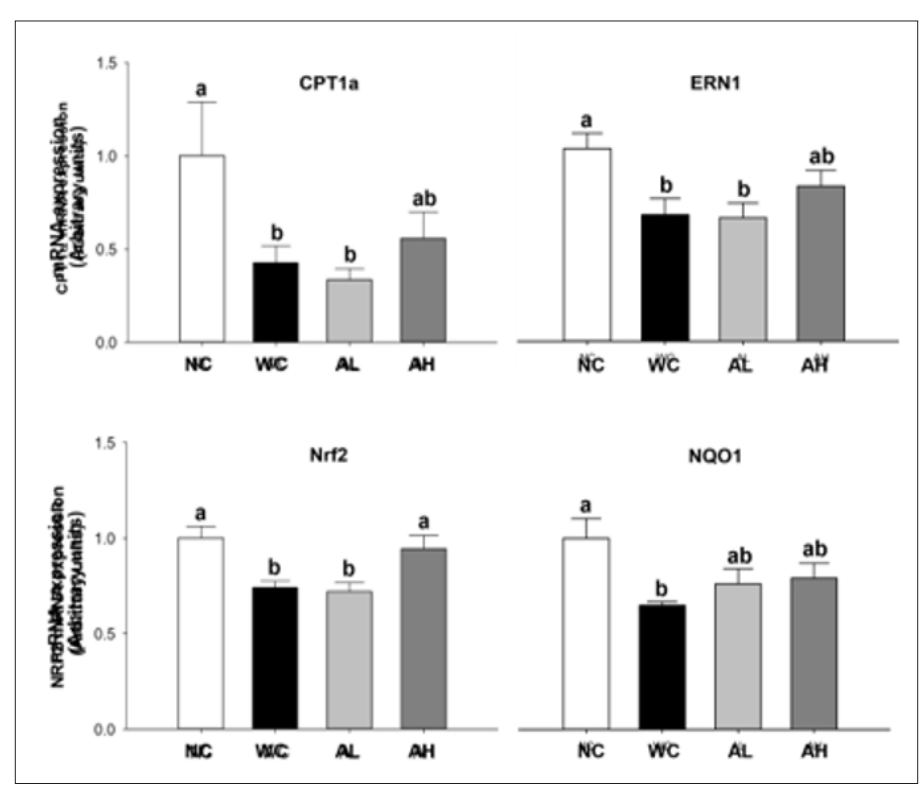

Figure 4: Gene expressions of CPT1区, ERN1, Nrf2, and NQO1 in the liver of C57BL/6J mice fed with WD and AK for 12 weeks. AK, Angelica keiskei juice powder; CPT1区, carnitine palmitoyltransferase II gene; ERN1, endoplasmic reticulum nuclei-1; $\mathrm{Nrf2}$, nuclear factor erythroid 2-related factor 2; NQO1, NAD $(\mathrm{P}) \mathrm{H}$ : quinone oxidoreductase 1; NC, normal control; WC, western diet control; AL, low-dose AK (2g/ kg BW) with WD; AH, high-dose AK (4 g/ kg BW) with WD. Data are represented as means \pm SEM ( $n=8$ /group). Values with different alphabets in each panel are significantly different at $P<0.05$ by Duncan's multiple comparison test.

\section{Discussion}

The goal of this study was to test the hypothesis that daily consumption of AK might attenuate the progression of hyperlipidaemia and hepatic steatosis caused by WD in male C57BL/6J mice. Protections against WD-induced destruction of intestinal barrier integrity and oxidative stress have been proposed as underlying mechanisms. Overall, our results support the hypothesis by demonstrating marked improvements in circulating and hepatic lipid profile, histological morphologies of the intestine and liver tissues, and gene expressions associated with ER oxidative stress in the group receiving high-dose AK compared with in the WD control group. Anatomically, the liver has a close interplay with the gut where nutrients and microbiome play major roles in the maintenance of healthy metabolism [21]. Previous studies have reported that a prototypic WD can cause alterations in microbial composition of the gut, leading to destruction of gut barrier integrity and liver damage [22]. In the present study, instead of changes in gut microbiota composition, we performed histopathological analysis to confirm changes in intestinal epithelial layers. To support morphological findings, we measured gene expressions of tight junction proteins (CLDN1 and OCLN) or mucin glycoprotein (MUC1).

Somewhat unexpectedly, mRNA expressions did not respond to WD or AK administration in this study. It might be because we used whole segment of small intestine rather than each segment of small intestine that may react differently in response to WD and/or AK treatments. In contrast, gene expression of LPL was significantly increased in the whole intestine of the WC group. This is consistent with previous results that showed WD-induced promotion of lipid absorption by suppressing Fiaf, a circulating inhibitor of LPL gene expression [23,24]. However, daily AK administration inhibited WD-induced increase in LPL mRNA expression. These results implicate the role of AK as an inhibitor of LPL to reduce lipid absorption in the intestine, thereby exacerbating hyperlipidaemia and TG deposition in the liver. This result is also in line with results of Qioa et al. [25], where resveratrol was suggested to inhibit lipid absorption by suppressing intestinal LDL mRNA expression in mice fed with a high-fat diet.

The ER is an essential organelle for the synthesis, folding, and modification of almost all endogenous proteins to be secreted or embedded in the plasma membrane [26]. Particularly, protein folding has a complex pathway, so that it is easy to be perturbed under physiological conditions like diet-induced oxidative stress [27]. The condition of an imbalance between ER protein load and folding capacity is referred to as "ER stress". Acute ER stress triggers an adaptive response known as "unfolded protein response (UPR)". However, prolonged or severe ER stress induces a defect 
in hepatic UPR signalling, resulting in the changes of metabolic gene regulations and the development of hepatic steatosis [28]. To understand the underlying action mechanisms of WD and AK, we determined ER stress-dependent gene expressions in mitochondria and microsome fractions of the liver. CPT $1 \alpha$ carries out the ratelimiting step in fatty acid $\beta$-oxidation in the mitochondria. ERN1 is an ER-resident transmembrane protein working as proximal sensor of hepatic UPR signalling, and thus important to prevent hepatic steatosis [29]. Nrf2 is a cytosolic protein involved in protecting cells against oxidative damage [30]. The ER can act as a sensor organelle through Nrf2 [31].

NQ01 is one of the best-characterized Nrf2 downstream genes [32]. The results of the present study showed that gene expressions of CPT1 $\alpha$, ERN1, Nrf2, and NQO1 were significantly decreased in WC group compared to those in NC group. These findings were supported by previous studies. Tanaka et al. [32] showed that HFD reduced mRNA expression of Nrf2 and its target genes, resulting in lipid accumulation and oxidative stress in the liver. Meakin et al. [33] have reported that, compared to wild type mice, mRNA expression levels of CPT $1 \alpha$ are lower in livers of Nrf2-KO mice. Taken together, these findings implicate the role of $\mathrm{AK}$ as an antioxidant substance in the liver. The UPLC-LTQ/MS analysis of AK also supported the potential antioxidant properties of AK. Coumarins and chalcones, signature chemical components of $\mathrm{AK}$, possess protective effects against ER oxidative stress [6,7].

When results of the present study are viewed collectively, the data demonstrated that high dose AK has a potential to decrease lipid delivery from intestine to the liver by both maintaining intestinal barrier integrity and decreasing intestinal LPL mRNA expression. In addition, high-dose AK exerted protective effect against ER oxidative stress in the liver, thereby reducing hepatic lipid accumulation. However, this study did not fully explain the underlying mechanisms of $\mathrm{AK}$ due to the following limitations. First, alterations of gut microbiota were not determined. Next, individual chemical components were not used to test which bioactive component was responsible for suppressing ER oxidative stress. Future studies are needed to explore how multi-components in AK produces synergistic effects by employing a comprehensive systems approach. However, even with these limitations, results and implications of this study are interesting and sufficient as scientific background to support the potential use of AK to control the onset and progression of NAFLD.

\section{Conclusion}

There might be multiple reasons why AK can prevent WDinduced hyperlipidemia and hepatosteatosis. In the present study, we specially focused on the role of AK in the gut-liver axis, demonstrating that $\mathrm{AK}$ could decrease lipid delivery to the liver by maintaining intestinal barrier integrity and decreasing LPL mRNA expression in the gut. Furthermore, we demonstrated that AK could improve plasma lipid levels, hepatic lipid accumulation, and inflammation by increasing fatty acid oxidation and reducing lipogenesis through restoration of ER-stress signaling. We also analyzed signature chemical components of AK, suggesting that coumarins and chalcones might be responsible bioactives. These findings suggest that daily consumption of AK juice may have potentials to prevent WD-induced NAFLD development through mitigating intestinal barrier damage, intestinal lipid absorption, and hepatic ER oxidative stress.

\section{Acknowledgement}

This study was supported by the Bio \& Medical Technology Development Program of the National Research Foundation (NRF) funded by the Ministry of Science \& ICT (2012M3A9C4048761).

\section{References}

1. Kil YS, Pham ST, Seo EK, Jafari M (2017) Angelica keiskei, an emerging medicinal herb with various bioactive constituents and biological activities. Arch Pharm Res 40(6): 655-675.

2. Okuyama T, Takata M, Takayasu J, Hasegawa T, Tokuda H, et al. (1991) Anti-tumor-promotion by principles obtained from Angelica keiskei. Planta Med 57(3): 242-246.

3. Zhang T, Yamashita Y, Yasuda M, Yamamoto N, Ashida H (2015) Ashitaba (Angelica keiskei) extract prevents adiposity in high-fat diet-fed C57BL/6 mice. Food \& function 6(1): 134-144.

4. Ohnogi H, Hayami S, Kudo Y, Deguchi S, Mizutani S, et al. (2012) Angelica keiskei extract improves insulin resistance and hypertriglyceridemia in rats fed a high-fructose drink. Biosci Biotechnol Biochem 76(5): 928932.

5. Matsuura M, Kimura Y, Nakata K, Baba K, Okuda H (2001) Artery relaxation by chalcones isolated from the roots of Angelica keiskei. Planta medica 67(03): 230-235.

6. Kim E, Choi J, Yeo I (2012) The effects of Angelica keiskei Koidz on the expression of antioxidant enzymes related to lipid profiles in rats fed a high fat diet. Nutr Res Pract 6(1): 9-15.

7. Yasuda M, Kawabata K, Miyashita M, Okumura M, Yamamoto N, et al. (2014) Inhibitory effects of 4-hydroxyderricin and xanthoangelol on lipopolysaccharide-induced inflammatory responses in RAW264 macrophages. J Agric Food Chem 62(2): 462-467.

8. Choi SH, Park KH (2011) Protective Effects of Angelica keiskei extracts against d-galactosamine (GalN)-induced hepatotoxicity in rats. Journal of Food Hygiene and Safety 26(3): 235-241.

9. Choi YH, Lee HS, Chung CK, Kim EJ, Kang IJ (2017) Protective effects of an ethanol extract of Angelica keiskei against acetaminophen-induced hepatotoxicity in HepG2 and HepaRG cells. Nutr Res Pract 11(2): 97-104.

10. Depner CM, Philbrick KA, Jump DB (2013) Docosahexaenoic acid attenuates hepatic inflammation, oxidative stress, and fibrosis without decreasing hepatosteatosis in a Ldlr(-/-) mouse model of western dietinduced nonalcoholic steatohepatitis. J Nutr 143(3): 315-323.

11. Cani PD, Bibiloni R, Knauf C, Waget A, Neyrinck AM, et al. (2008) Changes in gut microbiota control metabolic endotoxemia-induced inflammation in high-fat diet-induced obesity and diabetes in mice. Diabetes 57(6): 1470-1481.

12. Cotrim HP, Paraná R, Braga E, Lyra L (2000) Nonalcoholic steatohepatitis and hepatocellular carcinoma: natural history? The American journal of gastroenterology 95(10): 3018-3019.

13. Wheeler MD (2003) Endotoxin and Kupffer cell activation in alcoholic liver disease. Alcohol Res Health 27(4): 300-306.

14. Miura K, Kodama Y, Inokuchi S, Schnabl B, Aoyama T, et al. (2010) Tolllike receptor 9 promotes steatohepatitis by induction of interleukin1 beta in mice. Gastroenterology 139(1): 323-334.

15. Ishimoto T, Lanaspa MA, Rivard CJ, Roncal Jimenez CA, Orlicky DJ, et al. (2013) High-fat and high-sucrose (western) diet induces steatohepatitis that is dependent on fructokinase. Hepatology 58(5):1632-1643. 
16. Yilmaz B, Sahin K, Bilen H, Bahcecioglu IH, Bilir B, et al. (2015) Carotenoids and nonalcoholic fatty liver disease. Hepatobiliary surgery and nutrition 4(3): 161-171.

17. Qiu LX, Chen T (2015) Novel insights into the mechanisms whereby isoflavones protect against fatty liver disease. World Journal of Gastroenterology: WJG 21(4): 1099-1107.

18. Friedewald WT, Levy RI, Fredrickson DS (1972) Estimation of the concentration of low-density lipoprotein cholesterol in plasma, without use of the preparative ultracentrifuge. Clinical chemistry 18(6): 499502.

19. Folch J, Lees M, Sloane Stanley G (1957) A simple method for the isolation and purification of total lipids from animal tissues. J biol Chem 226(1): 497-509.

20. Kim DW, Curtis Long MJ, Yuk HJ, Wang Y, Song YH, et al. (2014) Quantitative analysis of phenolic metabolites from different parts of Angelica keiskei by HPLC-ESI MS/MS and their xanthine oxidase inhibition. Food Chem 153: 20-27.

21. Visschers RG, Luyer MD, Schaap FG, Olde Damink SW, Soeters PB (2013) The gut-liver axis. Curr Opin Clin Nutr Metab Care 16(5): 576-581.

22. Sheflin AM, Melby CL, Carbonero F, Weir TL (2017) Linking dietary patterns with gut microbial composition and function. Gut microbes 8(2): 113-129.

23. Van Olden C, Groen AK, Nieuwdorp M (2015) Role of Intestinal Microbiome in Lipid and Glucose Metabolism in Diabetes Mellitus. Clin Ther 37(6): 1172-1177.

24. Bäckhed F, Ding H, Wang T, Hooper LV, Koh GY, et al. (2004) The gut microbiota as an environmental factor that regulates fat storage. Proc Natl Acad Sci U S A 101(44): 15718-15723.

\section{ISSN: 2574-1241}

DOI: $10.26717 / B J S T R .2018 .10 .001933$

Oran Kwon. Biomed J Sci \& Tech Res

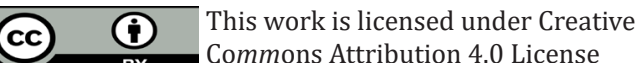

Submission Link: https://biomedres.us/submit-manuscript.php
25. Qiao Y, Sun J, Xia S, Tang X, Shi Y, et al. (2014) Effects of resveratrol on gut microbiota and fat storage in a mouse model with high-fat-induced obesity. Food \& function 5(6): 1241-1249.

26. Berridge MJ (2002) The endoplasmic reticulum: a multifunctional signaling organelle. Cell calcium 32(5-6): 235-249.

27. Digaleh H, Kiaei M, Khodagholi F (2013) Nrf2 and Nrf1 signaling and ER stress crosstalk: implication for proteasomal degradation and autophagy. Cell Mol Life Sci 70(24): 4681-4694.

28. Basseri S, Austin RC (2008) ER stress and lipogenesis: a slippery slope toward hepatic steatosis. Developmental cell 15(6): 795-796.

29.Zhang K, Wang S, Malhotra J, Hassler JR, Back SH, et al. (2011) The unfolded protein response transducer IRE1 $\alpha$ prevents ER stress-induced hepatic steatosis. The EMBO journal 30(7): 1357-1375.

30. Jaiswal AK (2004) Nrf2 signaling in coordinated activation of antioxidant gene expression. Free Radic Biol Med 36(10): 1199-1207.

31. Gorlach A, Klappa P, Kietzmann T (2006) The endoplasmic reticulum: folding, calcium homeostasis, signaling, and redox control. Antioxid Redox Signal 8(9-10): 1391-1418.

32. Tanaka Y, Aleksunes LM, Yeager RL, Gyamfi MA, Esterly N, et al. (2008) NF-E2-related factor 2 inhibits lipid accumulation and oxidative stress in mice fed a high-fat diet. Journal of Pharmacology and Experimental Therapeutics 325(2): 655-664.

33. Meakin PJ, Chowdhry S, Sharma RS, Ashford FB, Walsh SV, et al. ( 2014) Susceptibility of Nrf2-null mice to steatohepatitis and cirrhosis upon consumption of a high-fat diet is associated with oxidative stress, perturbation of the unfolded protein response, and disturbance in the expression of metabolic enzymes but not with insulin resistance. Mol Cell Biol 34(17): 3305-3320.

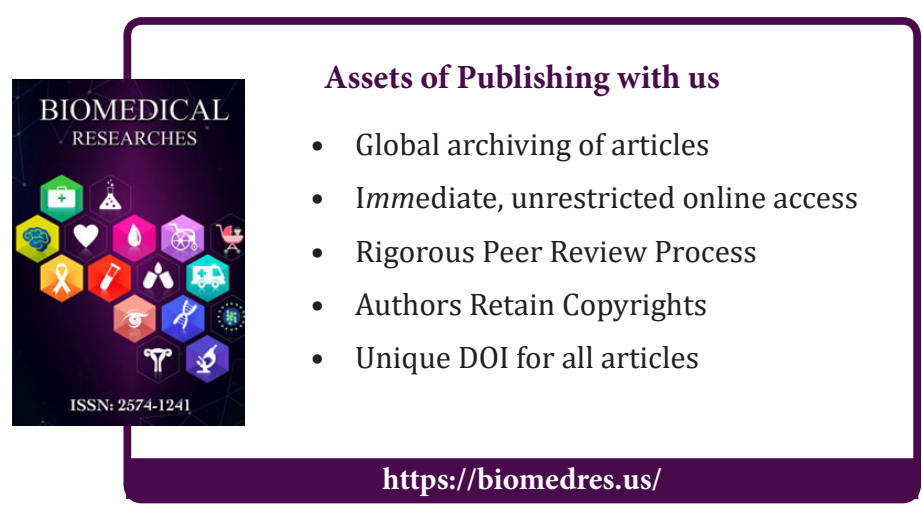

